\title{
EVOLUTION OF RAPIDLY ROTATING B-TYPE STARS
}

\author{
I. JULIANA SACKMANN* and S. P. S. ANAND \\ David Dunlap Observatory, University of Toronto, Canada
}

(Paper read by M. J. Clement)

\section{Introduction}

It has become clear in recent years through the work of Collins $(1963,1965,1966)$, Collins and Harrington (1966), Hardorp and Strittmatter (1968a, b) and Roxburgh and Strittmatter (1965) how seriously the spectrum of a star may be affected by rotation. However, before atmospheric models for rotating stars can be computed, the results of corresponding interior models have to be available. For polytropic configurations a great many rotating interior models have been constructed (Chandrasekhar, 1933; James, 1964; Stoeckly, 1965; Monaghan and Roxburgh, 1965; and Anand, 1968), but for rotating main-sequence stars few computations exist (Sweet and Roy, 1953; Roxburgh et al., 1965; Ostriker and Mark, 1968; Faulkner et al., 1968). In fact for B- and Be-type stars for which the most rapid rotation along the main sequence is observed, not a single interior model has yet been computed except in this conference reported by Kippenhahn and Thomas (1970). It is this gap that the present paper attempts to close.

Sixty-four interior main-sequence models of slowly and rapidly rotating B-type stars are presented. The mass of the models ranges from 5 to $15 M_{\odot}$ and the rotation is taken as uniform. The contribution of radiation pressure is fully included in the basic equations. The basic set of equations are solved by applying a Henyey type of iteration procedure as developed for non-rotating stars by Larson and Demarque (1964). The details of all these calculations are under publication (Sackmann and Anand, 1970).

\section{Results}

Tables Ia and Ib define the characteristics of the 64 main-sequence models. Table II gives some of the most important results for various models at zero and at critical rotation. Most of the parameters in these tables have the usual meanings (Sackmann and Anand, 1970).

\section{A. THE LUMINOSITY}

The luminosity as used here means the total bolometric interior luminosity. The decrease of this luminosity as the rotation increases when measured by the usual $\eta$-criterion, is displayed in Figure 1 for models of 5, 10, and $15 M_{\odot}$. The first feature to notice is the very close coincidence of the curves for the different mass models.

* Present address: Universitäts Sternwarte, Göttingen, Germany. 
TABLE Ia

Model characteristics

\begin{tabular}{lclcl}
\hline Model & $M / M_{\odot}$ & \multicolumn{2}{l}{ Chemical composition } & $\begin{array}{l}\text { Radiation } \\
\text { pressure }\end{array}$ \\
\cline { 5 - 5 } & & $X$ & $Z$ & included \\
I & 15 & 0.67 & 0.03 & included \\
II & 12 & 0.67 & 0.03 & included \\
III & 10 & 0.67 & 0.03 & included \\
IV & 9 & 0.67 & 0.03 & included \\
V & 7 & 0.67 & 0.03 & included \\
VI & 5 & 0.67 & 0.03 & omitted \\
VII & 5 & 0.67 & 0.03 & omitted \\
VIII & 5 & 0.71 & 0.02 &
\end{tabular}

TABLE Ib

Model characteristics

\begin{tabular}{lllllll}
\hline Model & $\alpha_{\mathrm{s}}$ & $\eta$ & $v$ & $R_{\mathrm{p}} / R_{\mathbf{0}}$ & $R_{\mathrm{e}} / R_{0}$ & $r_{i} / R_{0}$ \\
\hline 1 & 0 & 0 & 1.000 & 1.0000 & 1.0000 & 1.00 \\
2 & 0.05 & 0.0781 & 1.002 & 0.9756 & 1.0137 & 1.00 \\
3 & 0.10 & 0.1642 & 1.007 & 0.9524 & 1.0306 & 1.00 \\
4 & 0.15 & 0.2620 & 1.019 & 0.9302 & 1.0521 & 1.00 \\
5 & 0.20 & 0.3797 & 1.040 & 0.9091 & 1.0817 & 0.94 \\
6 & 0.25 & 0.5386 & 1.079 & 0.8889 & 1.1283 & 0.87 \\
7 & 0.30 & 0.9371 & 1.193 & 0.8696 & 1.2770 & 0.82 \\
8 & 0.300692 & 1.0000 & 1.200 & 0.8693 & 1.3040 & 0.82 \\
\hline
\end{tabular}

Although such a coincidence is observed for no other physical parameter, it must be related to the similarity of the structure of these stars.

The second feature to notice is that the maximum decrease in the luminosity due to rotation is extremely small, being only $6.7 \% \pm 0.1 \%$ for the models from 5 to $15 M_{\odot}$. This maximum decrease is much smaller than that of $24.7 \%$ obtained for critical rotation by Roxburgh et al. (1965) or that of $16.8 \%$ obtained for only slow rotation by Sweet and Roy (1953). Because they were the only ones available until recently, these two results have often been used in atmospheric calculations.

The third feature to notice is the detailed behavior of the luminosity change as the rotation builds up. This decrease in luminosity is certainly not linear with $\eta$. About $80 \%$ of the total change has already occurred when $\eta$ is only 0.5 of the critical value. A quadratic polynomial

$$
L(\eta)=L(0)\left[0.9977-0.1661 \eta+0.1028 \eta^{2}\right]
$$

is found to describe the $10 M_{\odot}$ curve better than $6 \%$.

\section{B. THE POLAR RADIUS}

The decrease of the polar radius with increasing rotation is displayed for the 64 mainsequence models in Figure 2. The first feature to notice is that a separation with mass occurs. The maximum percentage decrease at $15 M_{\odot}$ is only $1.2 \%$ while that of a 
EVOLUTION OF RAPIDLY ROTATING B-TYPE STARS

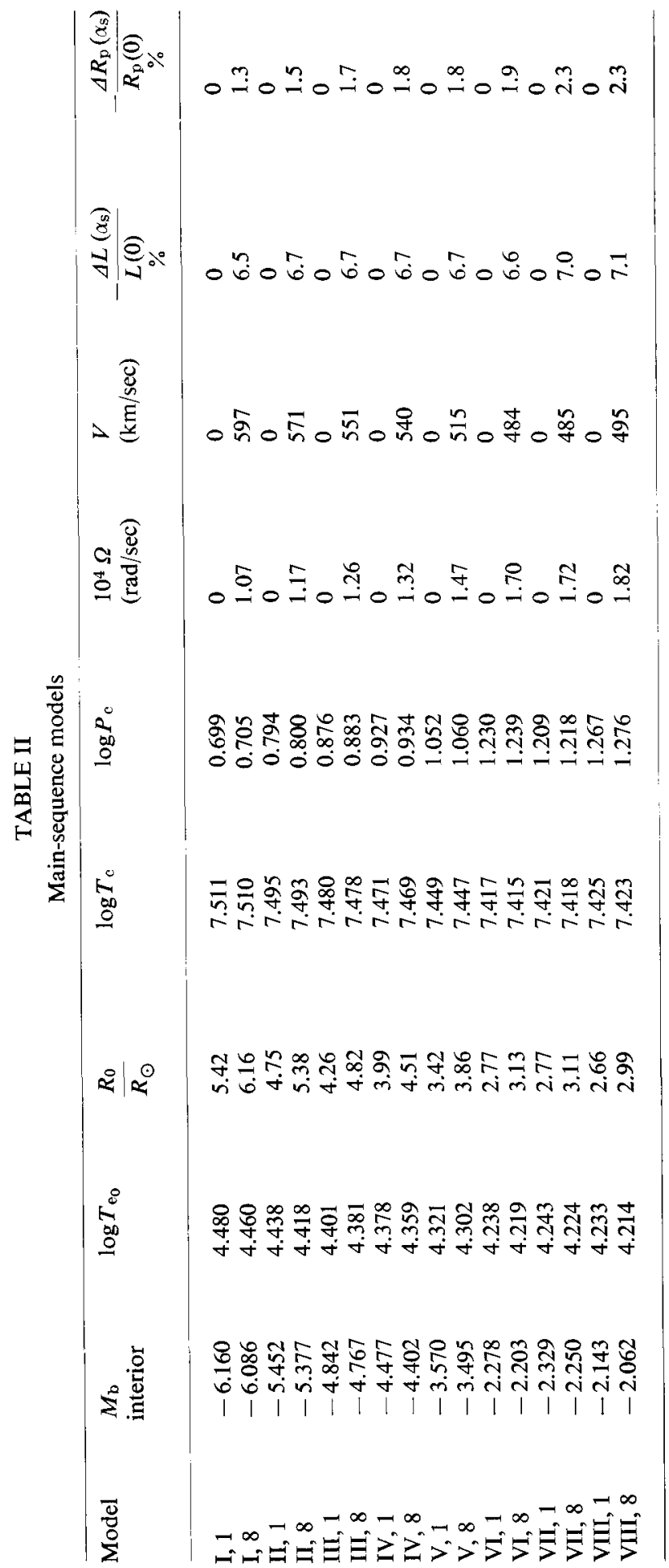




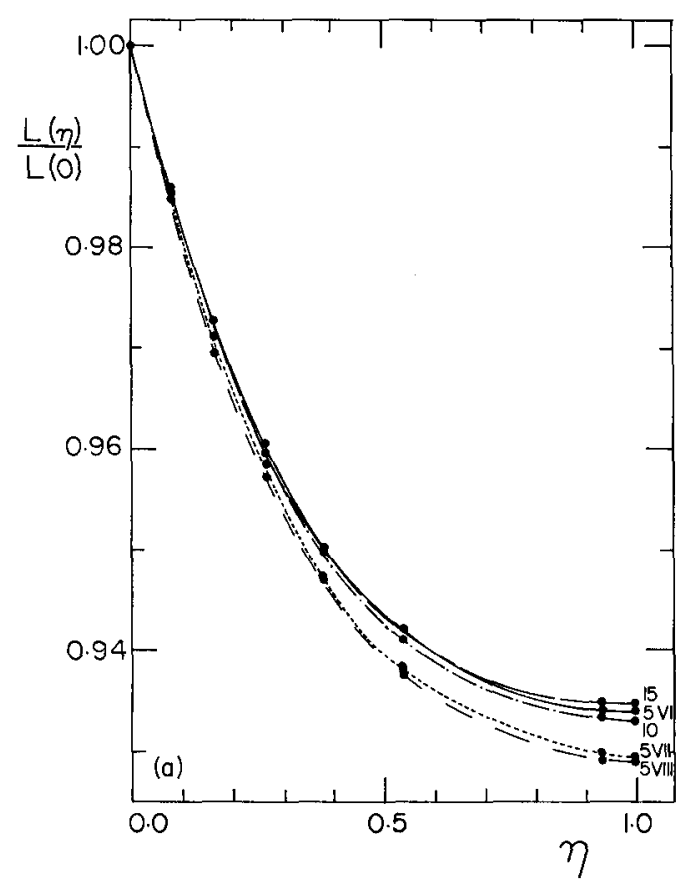

Fig. 1. The detailed variation, as the rotation builds up, of the luminosity of a rotating star relative to that of a non-rotating star of the same mass. The Roman numeral refers to the different models, while the Arabic numeral gives the mass of the models in solar units (reprinted courtesy of the Astrophysical Journal).

corresponding $5 M_{\odot}$ model is $1.9 \%$. Notice how small these changes are, especially when compared to those frequently used, namely those of Roxburgh et al. (1965), and Sweet and Roy (1953); the former obtained $10.87 \%$ at critical rotation and the latter $5.40 \%$ for slow rotation. Morover, the change of the polar radius when plotted against $\eta$ is highly non-linear. The quadratic polynomial

$$
R_{\mathrm{p}}(\eta)=R_{\mathrm{p}}(0)\left[0.9987-0.0486 \eta+0.0336 \eta^{2}\right]
$$

is found to describe the $10 M_{\odot}$ curve to better than $9 \%$.

The effects of rotation on other physical quantities are also investigated (Sackmann and Anand, 1970).

\section{THE EVOLUTION}

To allow for evolution, three modifications have to be applied to the basic structure equations given for main-sequence stars. The change of chemical composition as hydrogen is burned into helium is computed as in Larson and Demarque (1964). An additional release of energy due to gravitational contraction is included when the central-hydrogen content has fallen to a very low level. For rotating stars the change of the rotational velocity during evolution has to be considered as well. To compute 


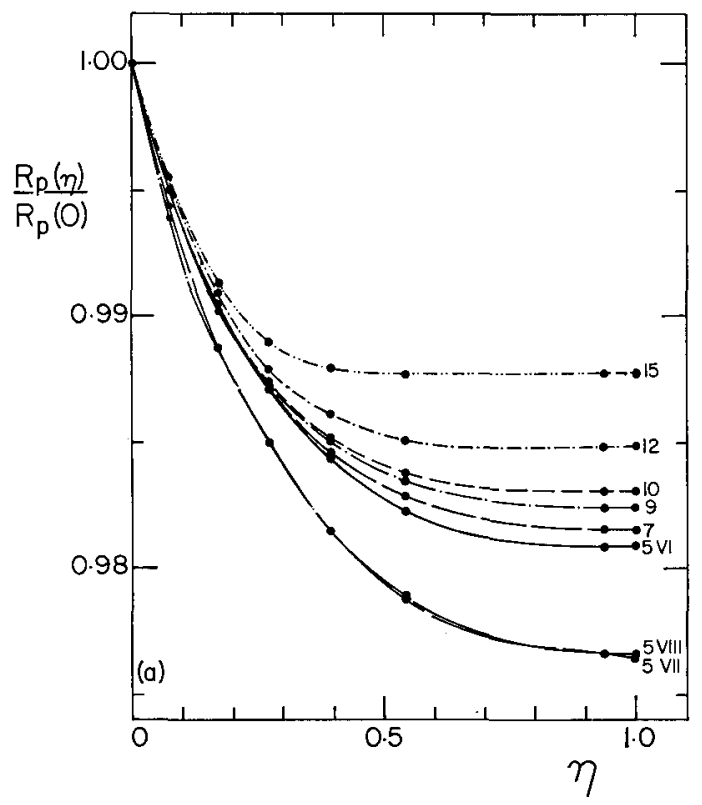

Fig. 2. The variation of the polar radius of a rotating star, relative to that of a non-rotating star having the same mass, as the rotation is increased. The Arabic and Roman numerals have the same meaning as in Figure 1 (reprinted courtesy of the Astrophysical Journal).

this change, one has to know whether angular momentum is conserved or lost during evolution. There are some recent observations by Kraft (1967) that suggest that latetype stars lose an appreciable amount of angular momentum on a time scale of $10^{8}$ years. However, since the present work is the first quantitative attempt to compute the evolutionary tracks of rotating stars, the case where angular momentum is conserved ought to be considered first.

There are two limiting cases in which angular momentum can be conserved. In the one case, angular momentum is conserved by cylindrical shells. This will give rise to differential rotation even if the star starts off rotating rigidly on the zero age mainsequence. The other limiting case is that in which the star as a whole, like a solid body, conserves its angular momentum. Thus, a star starting off with uniform rotation will continue to rotate uniformly during evolution. The latter case is of course the simplest and is considered in this work. The change in angular velocity with evolution is related to the change in the moment of inertia in an obvious way.

Because of the large amount of computer time needed, the evolution of only one mass was considered. A mass of $10 M_{\odot}$ was selected as being fairly representative of Be stars and also as being right in the middle of the 5 to $15 M_{\odot}$ range considered for main-sequence models. A number of evolutionary tracks for different initial rotational velocities were computed.

During evolution, the moment of inertia $(I)$ increases, causing angular velocity $(\Omega)$ to decrease if the star's total angular momentum is conserved. In Figure 3, the evo- 
lutionary change of the rotation parameters $\alpha_{s}$ and $\alpha_{c}$ and of the equatorial velocity $V$ and the angular velocity are shown.

It is very interesting to compare the equatorial velocities at critical rotation for evolved and main-sequence models. For a $10 M_{\odot}$ main-sequence model, the equatorial

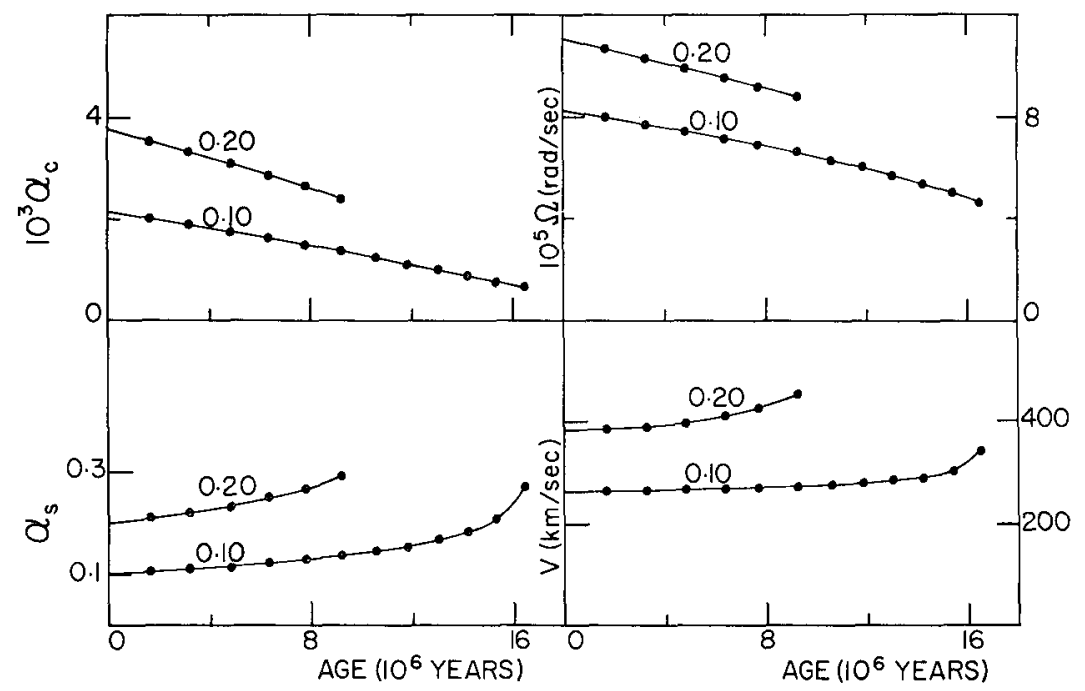

Fig. 3. For two rotating $10 M \odot$ models starting their evolution off the main-sequence with $\alpha_{\mathrm{s}}=0.10$ and $\alpha_{\mathrm{s}}=0.20$, respectively, the evolutionary change of the rotation parameters $\alpha_{\mathrm{s}}$ and $\alpha_{\mathrm{c}}$ and of the equatorial velocity $V$ and the angular velocity $\Omega$ are shown (reprinted courtesy of the Astrophysical Journal).

velocity at critical rotation is $551 \mathrm{~km} / \mathrm{sec}$, while for a $10 M_{\odot}$ evolved model with moderate initital rotation $\left(\sim \frac{1}{2}\right.$ critical) the equatorial velocity at critical rotation is only $344 \mathrm{~km} / \mathrm{sec}$. Hence, Be stars that are evolved could be expected to rotate with a critical equatorial velocity of the order of $200 \mathrm{~km} / \mathrm{sec}$ lower than that of corresponding main-sequence Be stars.

The most interesting result of the present computations are the evolutionary tracks of rotating stars plotted in the H-R diagram. Two evolutionary tracks of $10 M_{\odot}$ models rotating with different initial velocities are shown in Figure 4 together with the evolutionary track of the non-rotating model. Also illustrated are the main-sequences of non-rotating and critically rotating stars.

The evolutionary tracks of the two rotating stars are approximately parallel to each other and to the non-rotating case.

The last point plotted for the non-rotating model occurs just before the gravitational contraction phase. The last point plotted for the rotating models is the last output value from the computer before $\alpha_{\mathrm{s}}$ becomes greater than its critical value. The most interesting result of all the work here is that the evolved rotating models reach critical rotation long before gravitational contraction sets in unless the initial rotation is very slow. This behavior is in contrast to Schmidt-Kaler's (1964) suggestion, that the 


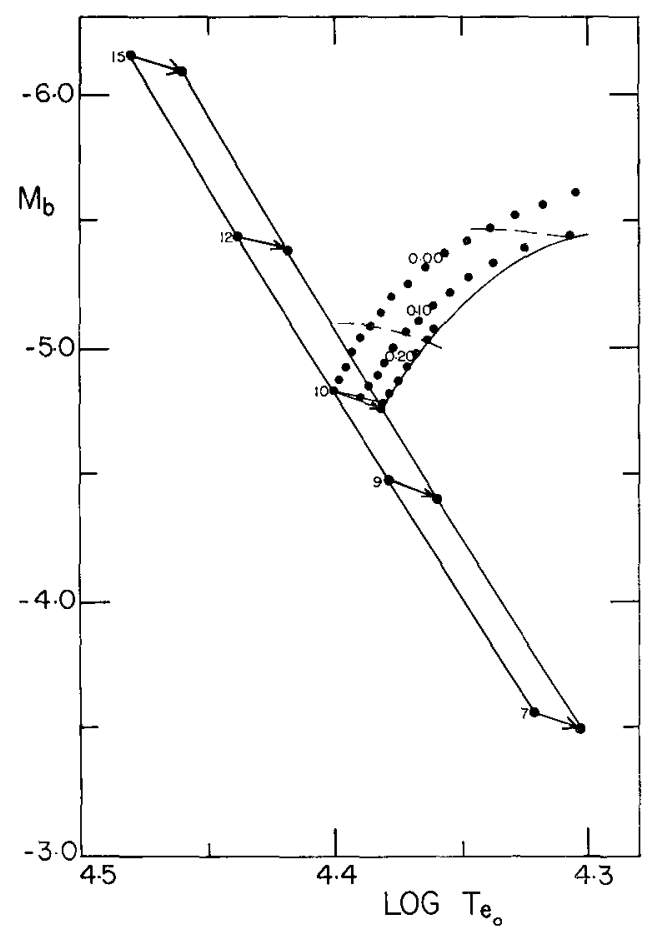

Fig. 4. The evolutionary tracks of $10 M_{\odot}$ models starting with different rotational velocities from the main-sequence. The number on a track refers to the initial $\alpha_{\mathrm{s}}$ value. The last point plotted for the tracks of rotating stars refers to the last output value from the computer before critical rotation sets in. The dashed curve refers to a locus of constant time. The solid curve to the right indicates the locus of $10 M_{\odot}$ stars at critical rotation. For comparison, the main-sequences of non-rotating and critically rotating stars are also shown. The numbers thereon refer to the masses in solar units (reprinted courtesy of the Astrophysical Journal).

extreme Be stars, sitting tightly grouped about a magnitude above the main-sequence in the $\mathrm{H}-\mathrm{R}$ diagram, are those rotating stars which are in their evolutionary stage just before, during, or immediately after the gravitational contraction phase. If SchmidtKaler's suggestion is indeed the correct one, then one must change the basic assumptions which led to the present evolved rotating models; that is, rotating stars either do not obey the momentum conservation law assumed here and/or they do not rotate uniformly. They would then be able to evolve up to the gravitational contraction phase before critical rotation sets in.

The time needed to reach critical rotation after the zero age mean sequence phase is $9.4 \times 10^{6}$ and $16.6 \times 10^{6}$ years for the models of initial $\alpha_{s}=0.20$ and $\alpha_{s}=0.10$, respectively.

The locus of evolved stars at critical rotation is also shown. This locus is very similar to the evolutionary tracks themselves.

Also interesting are the loci of constant time for the rotating models. These loci slope slightly upward and to the left in the H-R diagram. This is in contrast to the 
constant time locus obtained for non-rotating, evolved stars of different masses where the slope is upward to the right. Hence, an imaginary cluster consisting of rotating stars all of the same mass but having different initial rotational velocities would tend to show evolutionary effects by exhibiting a band of rapidly rotating stars parallel to the main-sequence and above it in accordance with age. Indeed Schild (1966), found for the $h$ and $\chi$ Persei cluster a band of ordinary Be stars, parallel and above the mainsequence, while the extreme Be stars occupy a much more localized section of this same band.

It should be pointed out that the evolved models computed here with zero rotation agree very well with independent evolutionary models of non-rotating stars. This gives some confidence in the evolutionary tracks of the rotating models.

\section{Conclusions and Suggestions}

Let us summarize the principal results of this investigation:

(1) The interior effect of uniform rotation on upper main-sequence stars is smaller than usually thought. For a given mass the maximum decrease in the interior luminosity is $6.7 \%$ and that of the polar radius by $1 \%$ to $2 \%$.

(2) The luminosity change due to rotation is remarkably constant as the mass varies from 5 to $15 M_{\odot}$; the corresponding polar radius change decreases as the mass increases.

(3) The assumption of a Roche model in the outer layers of a rotating star is a very good one, the distortion from a Roche model being no more than $0.05 \%$.

(4) At critical rotation, equatorial velocities vary from $484 \mathrm{~km} / \mathrm{sec}$ to $597 \mathrm{~km} / \mathrm{sec}$ and angular velocities from $1.70 \times 10^{-4}$ radians/sec to $1.07 \times 10^{-4}$ radians $/ \mathrm{sec}$ as the mass increases from 5 to $15 \mathrm{M}_{\odot}$.

(5) The evolution of rotating stars, computed by assuming conservation of total angular momentum and uniform rotation, causes the angular speed of rotation as well as the central rotation parameter $\alpha_{\mathrm{c}}$ to decrease. But the surface rotation parameter $\alpha_{\mathrm{s}}$ and the equatorial velocity increase with evolution. Critical equatorial velocities of evolved rotating stars can be of the order of $200 \mathrm{~km} / \mathrm{sec}$ smaller than those of corresponding main-sequence stars.

(6) The most important result found is that stars starting off from the main-sequence with a slow or intermediate rotation reach critical rotation very early in their evolution and still long before their gravitational contraction phase. Only for very slow initial rotation may a rotating star reach its gravitational contraction phase before critical rotation sets in. Schmidt-Kaler's (1964) suggestion that the extreme Be stars are evolved rotating stars in or close to their gravitational contraction phase can therefore not be supported with the present models. The present evolutionary track of a model with the rotation set equal to zero agrees well with that computed in independent work.

(7) The critical rotation locus in the H-R diagram is roughly parallel to the evolutionary tracks. 
(8) In the H-R diagram the locus of constant age for evolved rotating models of the same mass but with different initial rotational velocities has a slope of opposite sign to that produced by non-rotating stars differing in mass.

\section{Acknowledgements}

This research was supported in part by the National Research Council of Canada and the Department of University Affairs of the Province of Ontario.

Note added in proof: Recently Sanderson et al. (Astrophys. J. 159, L69, 1970) found the internal inconsistency in the calculation of the luminosity change by Roxburgh et al. (1965).

The corrected value of the luminosity change calculated by Sanderson et al. is in good agreement with the results of the present authors.

\section{References}

Anand, S. P. S.: 1968, Astrophys. J. 153, 135.

Chandrasekhar, S.: 1933, Monthly Notices Roy. Astron. Soc. 93, 390.

Collins, G. W., II: 1963, Astrophys. J. 138, 1134.

Collins, G. W., II: 1965, Astrophys. J. 142, 265.

Collins, G. W., II: 1966, Astrophys. J. 146, 914.

Collins, G. W., II and Harrington, J. P.: 1966, Astrophys. J. 146, 152.

Faulkner, J., Roxburgh, I. W., and Strittmatter, P. A.: 1968, Astrophys. J. 151, 203.

Hardorp, J. and Strittmatter, P. A.: 1968a, Astrophys. J. 151, 1057.

Hardorp, J. and Strittmatter, P. A.: 1968b, Astrophys. J. 153, 465.

James, R. A.: 1964, Astrophys. J. 140, 552.

Kippenhahn, R. and Thomas, H. C.: 1970, this volume, p. 20.

Kraft, R. P.: 1967, Astrophys. J. 150, 551.

Larson, R. B. and Demarque, P. R.: 1964, Astrophys. J. 140, 524.

Monaghan, J. J. and Roxburgh. I. W.: 1965, Monthly Notices Roy. Astron. Soc. 131, 13.

Ostriker, J. P. and Mark, J. W.-K.: 1968, Astrophys. J. 151, 1075 (Paper 1).

Roxburgh, I. W., Griffith, J. S., and Sweet, P. A.: 1965, Z. Astrophys. 61, 208.

Roxburgh, I. W. and Strittmatter, P. A.: 1965, Z. Astrophys 63, 15.

Sackmann, I. J. and Anand, S. P. S.: 1969, Astrophys. J. 155, 257.

Sackmann, I. J. and Anand, S. P. S.: 1970 (in press).

Schild, R. E.: 1966, Astrophys. J. 146, 142.

Schmidt-Kaler, Th.: 1964, Bonn. Veröffentl. 70, 1.

Stoeckly, R.: 1965, Astrophys. J. 142, 208.

Sweet, P. A. and Roy, A. E.: 1953, Monthly Notices Roy. Astron. Soc. 113, 701.

\section{Discussion}

Jordahl: What were the assumptions in the form of the equations of structure used to produce these models (i.e., the modifications to allow for rotation effects)?

Anand: In the basic structure equation, we have assumed that the system is rotating uniformly but neglected meridian circulation. The effect of radiation pressure is fully included in the basic equations. The opacity and the nuclear energy-generation formulae are those which have been used often for non-rotating stars. For more details, see Sackmann and Anand (1970, Astrophysical Journal, in press).

Roxburgh: What is the lowest rotational velocity on the main sequence that will give equatorial breakup during the nuclear evolution phase? 
Anand: $200 \mathrm{~km} / \mathrm{sec}$.

Roxburgh: This means that there is no problem with the Be stars as there will be many stars at equatorial breakup for a substantial fraction of their evolutionary lifetime so that the statistics on $\mathrm{Be}$ stars will be O.K.

Jaschek: But really the majority of the Be stars look slightly overluminous - mostly IV, sometimes even III.

Hardorp: (1) It has been said in the conclusion that the effects of rotation are smaller than hitherto thought: at critical velocity, luminosity, and polar radius drop only by $6 \%$ and $2 \%$, respectively, as compared to the non-rotating case. As far as the radius is concerned, this is a larger effect than found by Roxburgh et al., because it means an increase of the equatorial radius by $47 \%$, as compared to the $31 \%$ of these authors.

(2) For stars of 10 solar masses it has now been proven that they do reach critical rotational velocity already during their core hydrogen-burning phase, provided they start out faster than 250 $\mathrm{km} / \mathrm{sec}$ on the zero-age main sequence and rotation is always rigid. From the statistics of rotational velocities of the B2-B5 stars, P. Strittmatter and I concluded, by means of much rougher arguments, that most of the breakup stars are not created during this phase. The question whether these different answers are due to the fact that the B2-B5 stars have masses smaller than 10 solar masses and therefore show less change in moment of inertia, or whether our arguments are indeed too rough, could be settled by appelying Anand and Sackmann's computations to a star of 5 solar masses.

Deutsch: Miss Faber and Danziger have found strong observational evidence that most stars do evolve with uniform rotation. Can this evidence be dismissed as coincidental?

Slettebak: In this paper, as in the earlier paper by Hardorp and Strittmatter this afternoon, we have heard that $\mathrm{Be}$ stars are not expected to be located very far above the main sequence. Observations of Be stars in clusters and visual binary systems show that these objects are located in a band approximately one magnitude above the main sequence. Such displacements can be explained as an effect of rotation, according to the work of Collins and others. On the other hand, if one uses line ratios to estimate the luminosities of Be stars, the majority of the Be's seem to be of luminosity class IV and, in some cases, III. This is rather puzzling, in view of the recent results of Collins that rotation should not significantly affect the line ratios used to estimate luminosity, and suggests that the location of the Be stars on the H-R diagram is due in part to evolutionary effects in addition to aspect effects.

Collins: My conclusion relating to effects of rotation upon luminosity class are firm concerning stars of moderate to large rotational velocity. Concerning stars rotating with $\omega=\omega_{\mathrm{c}}$ the conclusions are more tentative but are still of a similar nature. If the Be stars are not rotating exactly at critical velocity what you say is true. If they are then the situation with regard to luminosity class may be much more complicated. 